\title{
HER3 intracellular domains play a crucial role in HER3/HER2 dimerization and activation of downstream signaling pathways
}

\author{
Byung-Kwon Choi ${ }^{1}$, Xiumei Cai ${ }^{1,2}$, Bin Yuan ${ }^{1,3}$, Zhao Huang $^{1}$, Xuejun Fan $^{1}$, Hui Deng ${ }^{1}$, Ningyan Zhang ${ }^{1 凶}$, \\ Zhiqiang $\mathrm{An}^{1 \Phi}$ \\ ${ }^{1}$ Texas Therapeutics Institute, Brown Foundation Institute of Molecular Medicine, University of Texas Health Science Center at \\ Houston, Houston, TX 77030, USA \\ 2 Department of Biochemistry and Molecular Biology, Shanghai Medical College, Fudan University, Shanghai 200032, China \\ 3 The Methodist Hospital Research Institute, Houston, TX 77030, USA \\ $\triangle$ Correspondence: zhiqiang.an@uth.tmc.edu (Z. An), ningyan.zhang@uth.tmc.edu (N. Zhang) \\ Received July 10, 2012 Accepted July 23, 2012
}

\begin{abstract}
Dimerization among the EGFR family of tyrosine kinase receptors leads to allosteric activation of the kinase domains of the partners. Unlike other members in the family, the kinase domain of HER3 lacks key amino acid residues for catalytic activity. As a result, HER3 is suggested to serve as an allosteric activator of other EGFR family members which include EGFR, HER2 and HER4. To study the role of intracellular domains in HER3 dimerization and activation of downstream signaling pathways, we constructed HER3/HER2 chimeric receptors by replacing the HER3 kinase domain (HER3-2-3) or both the kinase domain and the $\mathrm{C}$-terminal tail (HER3-2-2) with the HER2 counterparts and expressed the chimeric receptors in Chinese hamster ovary (CHO) cells. While over expression of the intact human HER3 transformed $\mathrm{CHO}$ cells with oncogenic properties such as AKT/ERK activation and increased proliferation and migration, $\mathrm{CHO}$ cells expressing the HER3-2-3 chimeric receptor showed significantly reduced HER3/HER2 dimerization and decreased phosphorylation of both AKT and ERK1/2 in the presence of neuregulin-1 (NRG-1). In contrast, CHO cells expressing the HER3-2-2 chimeric receptor resulted in a total loss of downstream AKT activation in response to NRG-1, but maintained partial activation of ERK1/2. The results demonstrate that the intracellular domains play a crucial role in HER3's function as an allosteric activator and its role in downstream signaling.
\end{abstract}

KEYWORDS HER3, HER2, cell proliferation, cell migration, PI3K/AKT, MAPK/ERK1/2

\section{INTRODUCTION}

The human epidermal growth factor receptor (EGFR or HER) family consists of four closely related type 1 transmembrane tyrosine kinase receptors: EGFR, HER2, HER3 and HER4 (Huang et al., 2009). EGFR, HER3 and HER4 exist in an inactive tethered (closed) confirmation and require ligand binding for activation, while HER2 has no reported ligand and exists in an open confirmation and is constitutively active for dimerization (Choi et al., 2012). HER receptors signal through the MAPK/ERK $1 / 2$ and PI3K/AKT pathways and form a signaling network which functions in concert with complex compensatory and feedback regulatory loops (Huang et al., 2009). Aberrant activation of HER receptors can tip the balance and cause deteriorative effects on cellular events that lead to various diseases such as cancer (Shepard et al., 2008). Among all the homodimer and heterodimer pairs, HER3/HER2 is the most potent partner for activation of the PI3K/AKT signaling cascade through direct HER3 binding to the p85 subunit of PI3K (Mattoon et al., 2004; Suenaga et al., 2005; Amin et al., 2010b). Growing evidence suggests that HER3 plays a pivotal role in regulation of the HER signaling cascade and it is emerging as a cancer drug target (Engelman et al., 2007; Kong et al., 2008; Wheeler et al., 2008; Baselga and Swain, 2009; Narayan et al., 2009; 
Schoeberl et al., 2009; Amin et al., 2010a).

Dimerization among the EGFR family of receptors leads to allosteric activation of the kinase domains of the partners, and the activated kinase domains in turn phosphorylate the tyrosine residues in the C-terminus of the receptors (Jura et al., 2009b; Jura et al., 2009a). HER3 is different from EGFR, HER2 and HER4 in that it lacks intrinsic kinase activity and has not been reported to form homodimers on cell surface (Sierke et al., 1997; Berger et al., 2004). Since the catalytically inactive kinase domain of HER3 is not capable of activating the kinase domain of its partnering receptors, HER3 is suggested to have evolved as a dedicated and specialized activating receptor in the EGFR family (Jura et al., 2009a; Amin et al., 2010a).

Despite the significant role that HER3 plays in cell signaling and cancer development, our understanding of the HER3 biology remains limited. Little is known about the role that HER3 intracellular domains play in dimerization with other HER family receptors and activation of downstream signaling. In this study, we constructed HER3/HER2 chimeric receptors by replacing the HER3 intracellular domains with their HER2 counterparts and over expressed the chimeric receptors in $\mathrm{CHO}$ cells to investigate the effect of intracellular domains on HER3/HER2 dimerization and downstream signaling.

\section{RESULTS}

\section{Expression of human wild type HER3 and chimeric HER3/HER2 in CHO cells}

To investigate the role of intracellular domains on HER3 function, we engineered two HER3/HER2 chimeric constructs: HER3-2-2 and HER3-2-3. The HER3-2-2 construct has the HER3 TK and CTD domains replaced with the HER2 corresponding region (AA712-1255) (Fig. 1A). The HER3 TK domain was replaced with the HER2 TK domain in the HER3-2-3 construct (AA712-990) (Fig. 1A). Stable CHO cell lines which express the wild type HER3 and the two HER3/HER2 chimeric constructs were developed. Endogenous EGFR expression was not detectable, but various levels of endogenous HER2, HER3 and HER4 expression were detected in $\mathrm{CHO}$ cells by RT-PCR (Fig. 1B). CHO cells that stably express the intact HER3 and HER3/2 chimeras showed significantly enhanced HER3 expression as indicated by Western blotting and flow cytometry (Fig. 1C and 1D). These results suggest that replacing HER3 TK and CTD domains with the HER2 counterparts did not affect HER3 protein expression. Similarly, we measured endogenous HER2 expression on CHO-HER3 and HER3/2 chimera cells in comparison with CHO-pcDNA cells, and the results showed that over expression of human HER3 and HER3/HER2 chimeras did not impact the endogenous HER2 expression levels (Fig. 1E and 1F).
Human HER3 readily interacts with CHO HER2, but HER3/2 chimeras show impaired heterodimerization with HER2

We performed co-immunoprecipitation (co-IP) to study the ability of human HER3 and the HER3/2 chimeras to heterodimerize with the endogenous HER2 in $\mathrm{CHO}$ cells. Consistent with the flow cytometry detection data shown in Fig.1E, total HER2 expression is comparable in all the cell lines tested and the level of expression is independent of NRG-1 stimulation (Fig. 2A, top panel). However, in the HER3 co-IP samples, NRG-1 stimulation significantly enhanced both phosphorylated HER2 (pHER2) and phosphorylated HER3 (pHER3) in the CHO-HER3 cells (Fig. 2A, bottom panel). In contrast, the HER3 co-IP complex from CHO-HER3-2-3 chimera cells showed much reduced pHER2, but pHER3 activation was still evident in this chimera (Fig. 2A, bottom panel). There was no detectable pHER2 or pHER3 in the HER3 co-IP complex from CHO-HER3-2-2 cells, even though HER3-2-2 receptor protein expression was comparable to the level of CHO-HER3 protein (Fig. 2A, bottom panel). It is noted that a significant basal level of pHER3 independent of NRG-1 stimulation is present in CHO-HER3 (Fig. 2A, bottom panel).

To further investigate the effect of HER3 TK and CTD domains on HER3 functions, we detected in situ dimerization of HER3 and HER2 using a fluorescence imaging method known as the proximity ligation assay (PLA). As shown in Fig. 2B, significant levels of HER2/HER3 heterodimers were present on the CHO-HER3 cell surface in response to NRG-1 stimulation. No HER2/HER3 dimers were detected on CHO-HER3-2-2 cells, and much reduced levels of HER2/HER3 dimers were observed on CHO-HER3-2-3 cells (Fig. 2B). These results are consistent with findings from the co-IP study that the HER3 intracellular domains TK and CTD are critical for HER2/HER3 interaction. Replacement of both the intracellular TK and CTD domains abolished HER3/HER2 interaction and replacement of the TK domain alone severely reduced the ability of the HER3 to interact with HER2.

\section{The HER3 kinase domain and the C-terminus domain are required for HER3/HER2 mediated $\mathrm{CHO}$ cell proliferation and migration}

Over expression of HER3 in CHO-HER3 cells significantly enhanced cell proliferation in the presence of NRG-1 stimulation compared to the parental $\mathrm{CHO}$ control cells (Fig. 3A). Proliferation in response to NRG-1 was also increased significantly in CHO-HER3-2-3 cells but to a lesser extent than in CHO-HER3 cells (Fig. 3A). In contrast, HER3-2-2 cells did not respond to NRG-1 stimulation (Fig. 3A).

We further investigated whether HER3 over expression and activation can affect $\mathrm{CHO}$ cell migration in response to NRG-1 in a trans-well migration assay. Compared to parental $\mathrm{CHO}$ cells, $\mathrm{CHO}-\mathrm{HER} 3$ cells showed increased migration at 
A

\begin{tabular}{|c|c|c|c|}
\hline \multicolumn{4}{|c|}{ Signal peptide (1-19) } \\
\hline \multirow[t]{2}{*}{ HER3 } & ECD (20-643) & TK (683-978) & CTD (979-1342) \\
\hline & & 961 & 1226 \\
\hline \multirow[t]{2}{*}{ HER3-2-2 } & ECD (20-643) & HER2 TK (712-990) & HER2 CTD (991-1255) \\
\hline & & 961 & 1325 \\
\hline HER3-2-3 & ECD (20-643) & HER2 TK (712-990) & HER3 CTD (979-1342) \\
\hline
\end{tabular}

B

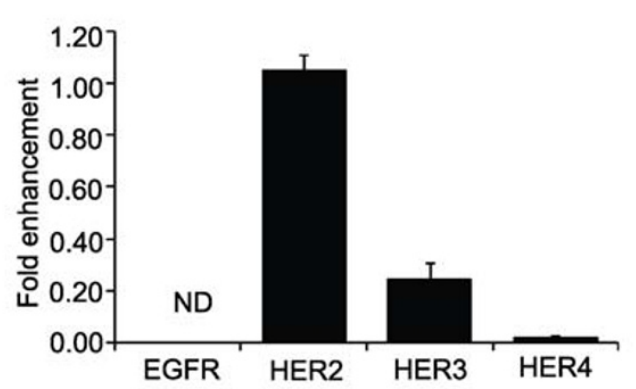

C

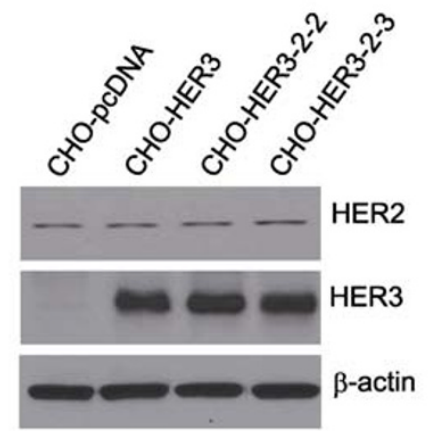

D

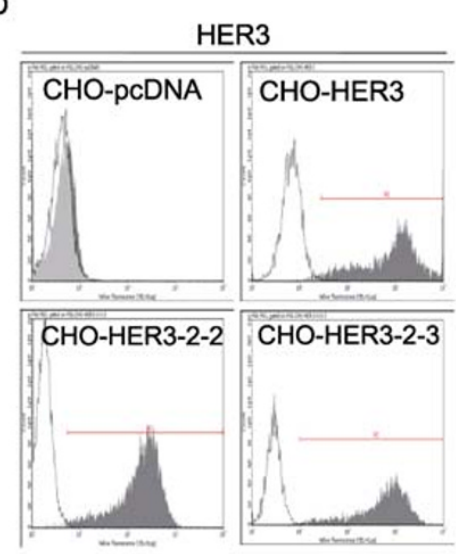

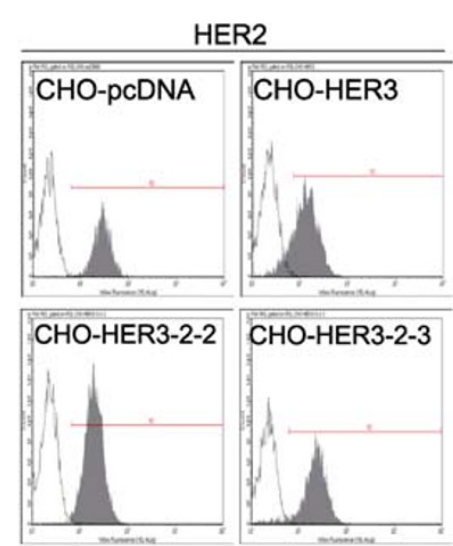

F

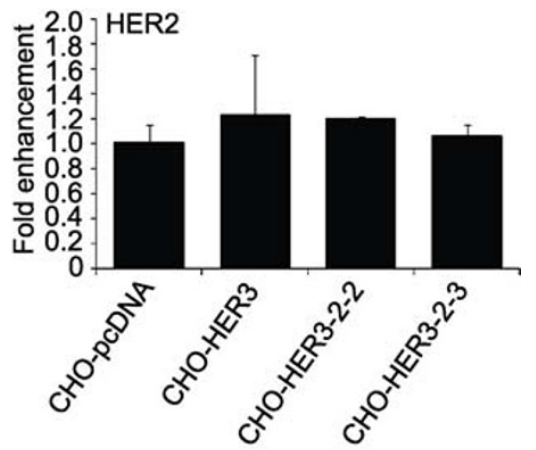

Figure 1. Construction of HER3/HER2 chimeric receptors and their expression in CHO cells. (A) Schematic diagram of HER3 and engineered HER3/2 chimeric receptors containing sub-domains of HER2. Numbers of each domain indicated original amino acid residue positions in HER2 and HER3 protein templates. (B) Endogenous EGFR, HER2, HER3 and HER4 expression in $\mathrm{CHO}$ cells as determined by qPCR. mRNA levels were normalized to the housekeeping gene, $\beta$-actin, and presented as fold enhancement compared to mRNA level of each gene in CHO-pcDNA at basal state. ND, not detectable. (C) Endogenous expression of HER2 and heterologous expression of HER3, HER3-2-2, and HER3-2-3 in CHO as determined by Western blotting. (D) Endogenous expression of HER3 and heterologous expression of HER3, HER3-2-2, and HER3-2-3 in CHO as determined by flow cytometry. (E) Endogenous HER2 expression in wild type $\mathrm{CHO}$ cells and $\mathrm{CHO}$ cells stably expressing HER3, HER3-2-3, and HER3-2-2 as measured by flow cytometry. (F) Endogenous expression of HER2 in wild type $\mathrm{CHO}$ cells and $\mathrm{CHO}$ cells stably expressing HER3, HER3-2-3, and HER3-2-2 as determined by qPCR.

basal state and significantly enhanced migration by NRG-1 stimulation (Fig. 3B). Replacement of the HER3 kinase domain alone with the HER2 counterpart in CHO-HER3-2-3 cells reduced the effect on migration, a result consistent with the partial activation of pHER3 in this construct (Fig. 3B). However, replacement of both the intracellular TK and CTD domains with HER2 counterparts abolished the effects of HER3 mediated cell migration in response to NRG-1 (Fig. 3B).
Activation of MAPK/ERK pathway through HER3/HER2 dimerization is linked to both HER3 and HER2 intracellular domains, but PI3K/AKT signaling is primarily driven by the HER3 C-terminal tail

In CHO-HER3 cells, both HER2 and HER3 phosphorylation were driven by NRG-1 stimulation (Fig. 4A). In CHO-HER32-3 cells, HER3 phosphorylation was also stimulated by 
A
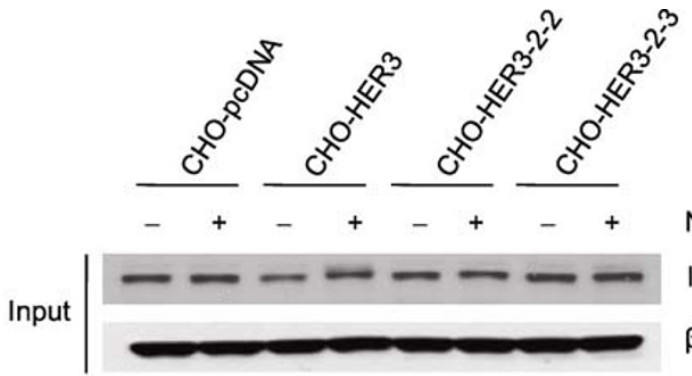

NRG-1, $100 \mathrm{ng} / \mathrm{mL}$

IB: HER2

$\beta$-actin

IP: HER3

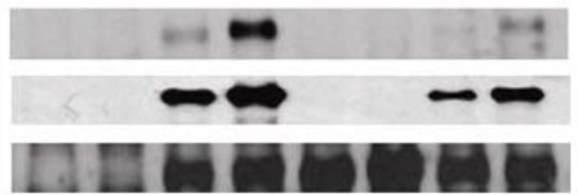

IB: pHER2 (Y1221)

IB: pHER3 (Y1289)

IB: HER3

B
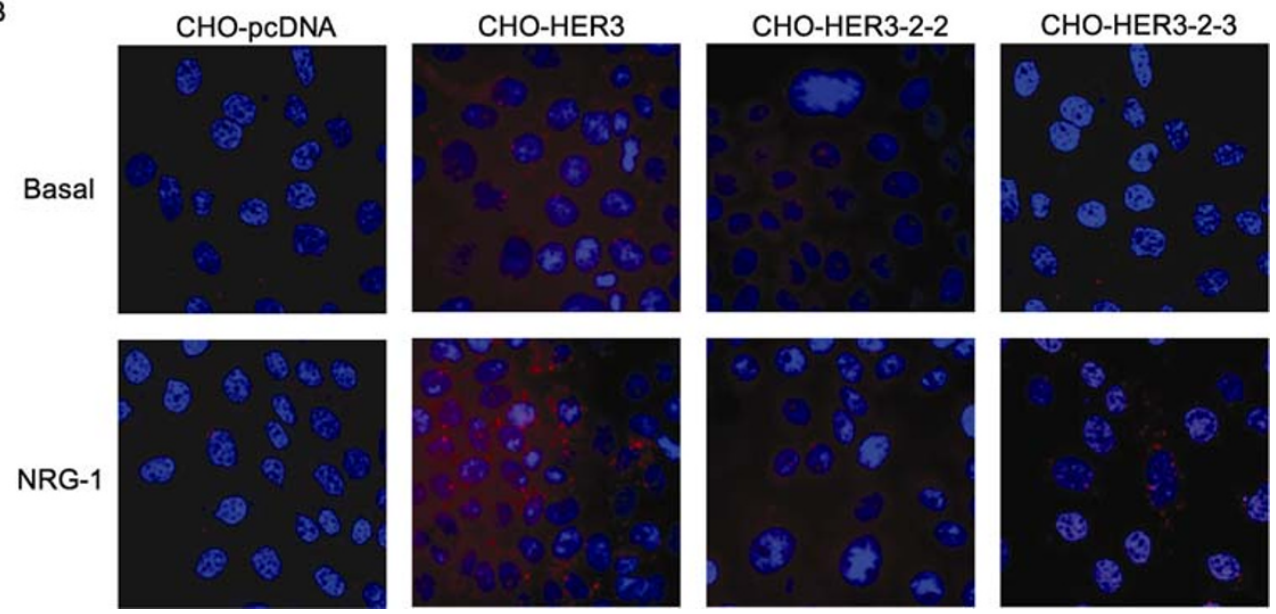

Figure 2. Effect of replacing HER3 TK and CTD with HER2 counterparts on HER2 and HER3 phosphorylation and heterodimerization. (A) Top panel: total HER2 expression in wild type $\mathrm{CHO}$ cells and $\mathrm{CHO}$ cells stably expressing HER3, HER3-2-3, and HER3-2-2 as measured by Western blot. Bottom panel: Western blot detection of total HER2, phospho-HER2 and phospho-HER3 in immunoprecipitated complex by an anti-HER3 antibody, HER3mAb. (B) Visualization of HER2 and HER3 heterodimerization by the proximity ligation assay (PLA) using mouse anti-HER3 antibody and rabbit anti-HER2 antibody.

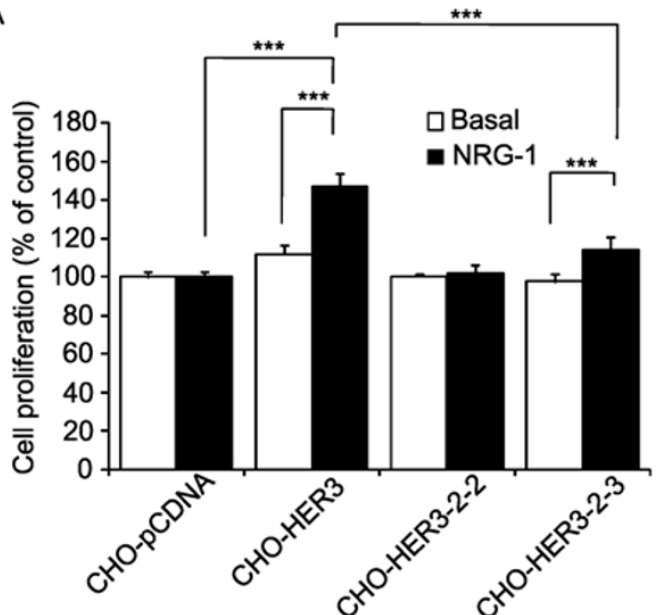

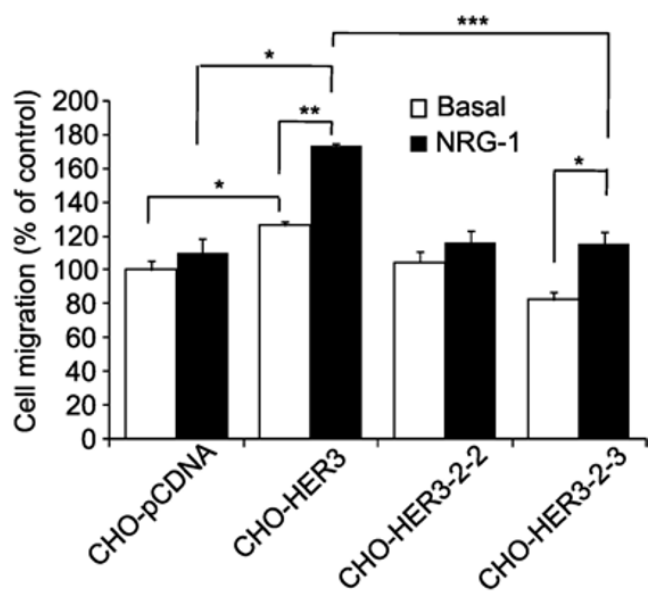

Figure 3. Effect of replacing HER3 TK and CTD with HER2 counterparts on cell proliferation and migration. (A) Cell proliferation of $\mathrm{CHO}, \mathrm{CHO}-\mathrm{HER} 3, \mathrm{CHO}-\mathrm{HER} 3-2-2$ and $\mathrm{CHO}-\mathrm{HER} 3-2-3$ cells in the absence and presence of NRG-1 stimulation. (B) Cell migration of $\mathrm{CHO}$, CHO-HER3, CHO-HER3-2-2 and CHO-HER3-2-3 cells in the absence and presence of NRG-1 stimulation. Student T-test was applied for pair wise statistical comparisons between treatments, ${ }^{*}=P<0.05,{ }^{* *}=P<0.01$, and ${ }^{* \star *}=P<0.001$. 
NRG-1, but the pHER3 signal is much reduced when compared with the wild type CHO-HER3 cells (Fig. 4A). However, pHER2 in CHO-HER3-2-3 cells was not detectable (Fig. 4A).

In CHO-HER2 cells which over express the human HER2, HER2 phosphorylation is dependent on NRG-1 stimulation. In contrast, constitutive HER2 phosphorylation was detected in HER3-2-2 cells without NRG-1 stimulation (Fig. 4B). Even though HER3-2-2 is abundantly expressed in CHO-HER3-2-2 cells, HER3 phosphorylation is not expected since the HER3 C-terminal tyrosine tail is replaced with the HER2 counterpart (Fig. 4B).

To investigate how HER2 and HER3 over expression affects the downstream signaling in $\mathrm{CHO}, \mathrm{CHO}-\mathrm{HER} 2$, CHO-HER3, CHO-HER3-2-2 and CHO-HER3-2-3 cells, we monitored phosphorylation status of AKT and ERK1/2. Consistent with NRG-1 dependent HER2 and HER3 phosphorylation, pAKT was detected in both CHO-HER3, CHO-HER32-2 and CHO-HER3-2-3 cells in response to NRG-1 stimulation; with significantly reduced levels in CHO-HER3-2-2 and CHO-HER3-2-3 cells (Fig. 4C). These results suggest that $\mathrm{PISK} / \mathrm{AKT}$ signaling is primarily driven by the HER3 C-terminal regulatory domain. NRG-1 also stimulated ERK1/2 phosphorylation in CHO-HER3 cells, but NRG-1 stimulated ERK1/2 phosphorylation in CHO-HER3-2-2 and CHO-HER32-3 cells was much reduced (Fig. $4 \mathrm{C}$ ), suggesting that the HER3 kinase domain is required for ERK1/2 activation. Constitutive ERK1/2 phosphorylation was detected in CHO-HER2 cells (Fig. 4C). AKT phosphorylation was induced in CHO-HER3-2-2 cells upon NRG-1 stimulation, but not in CHO-HER2 cells (Fig. 4C).

\section{DISCUSSION}

Unlike HER2 and EGFR, the biology of HER3 activation in cancer development is less understood. Recent evidence from both basic and clinical studies suggests that HER3 serves as a signaling hub for the EGFR family of receptors (Amin et al., 2010a, b; Campbell et al., 2010). In this study, we employed a heterologous expression system to over express human HER3 and HER3/HER2 chimeric receptors in $\mathrm{CHO}$ cells to examine the role of intracellular domains of HER3 in heterodimerization and HER3 downstream signaling. $\mathrm{CHO}$ cells are known to not express endogenous EGFR (Krug et al., 2002). This is confirmed in the $\mathrm{CHO}$ cells used in our study by RT-PCR. In addition to the lack of EGFR expression, HER4 expression was minimally detected. It has been reported that HER2 expression is not detectable by Northern blotting in $\mathrm{CHO}$ cells (Chan et al., 1995), but we were able to detect low levels of HER2 expression by both RT-PCR and flow cytometry. HER3 expression in $\mathrm{CHO}$ cells was minimally detected by RT-PCR and flow cytometry. As expected, CHO-HER3 showed high levels of HER3 expression as indicated by both RT-PCR and flow cytometry. The lack of detectable expression of EGFR and minimal expres-
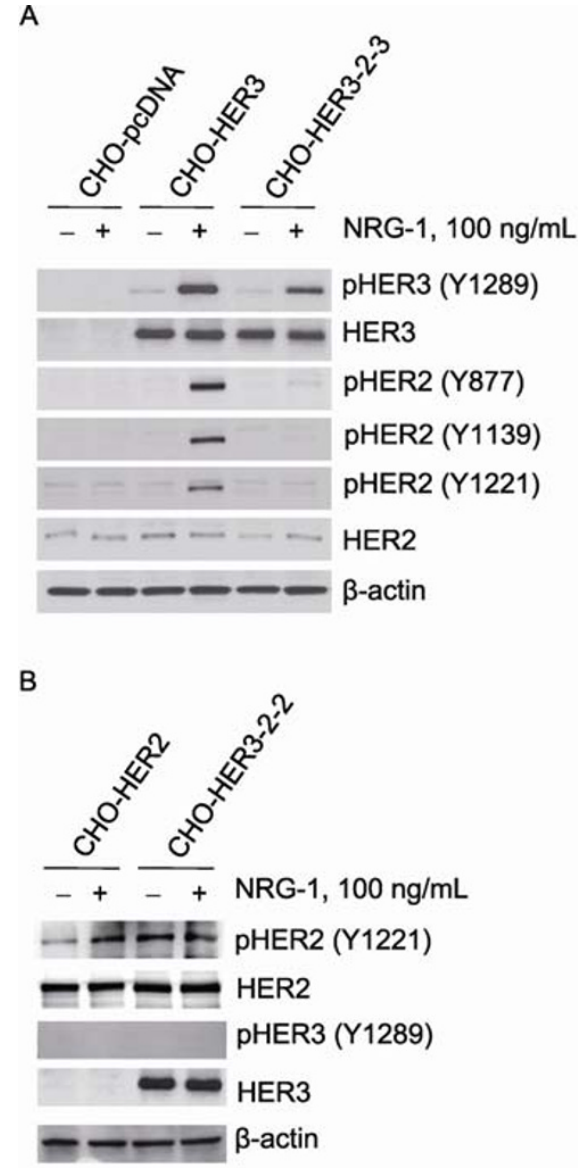

C

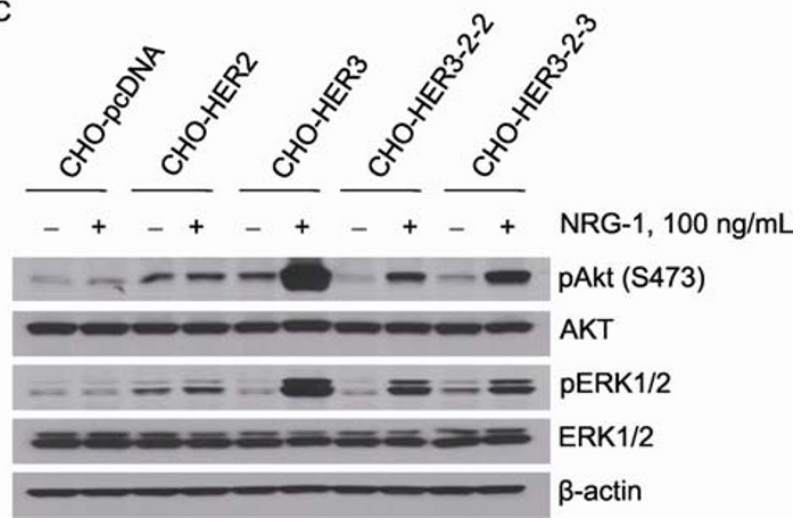

Figure 4. Effect of replacing HER3 TK and CTD with HER2 counterparts on AKT and ERK1/2 signaling pathways. (A) HER2 and HER3 phosphorylation in CHO-pcDNA, CHO-HER3 and CHO-HER3-2-3 cells in the absence and presence of NRG-1 stimulation. (B) Phosphorylation of HER3 and HER2 in CHO-HER2 and CHO-HER3-2-2 cells in the absence and presence of NRG-1 stimulation. (C) Phosphorylation of ERK1/2 and AKT in CHO-pcDNA, CHO-HER2, CHO-HER3, CHO-HER3-2-2 and CHO-HER3-2-3 cells in the absence and presence of NRG-1 stimulation. $\beta$-actin serves as loading control. 
sion of HER3 and HER4 make $\mathrm{CHO}$ a suitable model system to study HER3/HER2 interaction by expressing human HER3 in $\mathrm{CHO}$ cells. Our study showed that the human HER3 can readily heterodimerize with the endogenous $\mathrm{CHO}$ HER2. In addition, HER3 $3_{\text {human }} / \mathrm{HER} 2_{\mathrm{CHO}}$ dimerization in $\mathrm{CHO}$ cells is functional in response to NRG-1 stimulation, and activation of phosphorylation of HER2, HER3 and downstream signaling pathways such as PI3K/AKT and ERK1/2 (Figs. S1 and S3). More importantly, over expression of human HER3 in $\mathrm{CHO}$ cells mediated cell proliferation and migration (Fig. S1). It is interesting to note that NRG-1 had no effect on proliferation of control vector transfected $\mathrm{CHO}$ cells or HER2 phosphorylation, but the dual EGFR/HER2 tyrosine kinase inhibitor lapatinib inhibited wild type $\mathrm{CHO}$ proliferation independent of NRG-1 proliferation (Fig. S1). These results suggest that homodimerization of endogenous HER2 and heterodimeriztion of endogenous HER2 and HER3 are responsible to proliferation which is mediated by constitutive and basal cellular signaling independent of ligand stimulation. We further validated that the enhanced proliferation of $\mathrm{CHO}-\mathrm{HER} 3$ cells was the result of activation of PI3K/AKT signaling because the PI3K inhibitor wortmannin can neutralize the NRG-1 induced cell proliferation of CHO-HER3 cells (Fig. S2A). HER3 and the p85 subunit of PI3K co-IP studies confirmed that activation of HER3 by NRG-1 increased association with p85 in CHO-HER3 cells (Fig. S2B). However, the association of HER3 with p85 in CHO-HER3-2-2 cells and in CHO-HER3-2-3 cells was disrupted and decreased, respectively (Fig. S2B). In addition, wortmannin had no effect on the phosphorylation of HER2 and HER3, but suppressed NRG-1 stimulated AKT phosphorylation (Fig. S2C). Although NRG-1 stimulated phosphorylation of MAP kinases in CHO- HER3 cells (Fig. S2C), wortmannin did not affect pERK1/2 suggesting that activation of MAPK signaling pathway is mediated through HER2/HER3 interaction and HER3 plays a more direct role in PI3K/AKT pathway activation. Collectively, these results suggest that HER3/HER2 dimerization can activate both PI3K/AKT and MAPK signaling pathways with HER3 activation directly engaging the PI3K/AKT pathway and HER2 in the HER3/HER2 pair engaging MAPK pathway activation. This is the first report that over expressing $\mathrm{HER} 3_{\text {human }}$ can transform a mammalian cell line such as $\mathrm{CHO}$ to possess cancer cell hallmarks which includes proliferation and migration.

In comparison with the wild type HER3, replacement of the HER3 intracellular TK domain with the HER2 counterpart reduced dimerization between HER2 and HER3 as measured by the PLA assay for extracellular domain association and co-IP for association of HER2 and HER3-2-3 molecules. The reduced dimerization was reflected by decreased phosphorylation of HER2, HER3, AKT and MAPK/ERK1/2. The reduced HER3/HER2 dimerization was further manifested in decreased cell proliferation and migration in CHO-HER3-2-3 cells. Despite the significant reduction, HER3-2-3 is able to dimerize with HER2 as indicated by the co-IP and PLA assays.

Replacement of both kinase domain (TK) and C-terminal tail (CTD) of HER3 with HER2 counterparts blocked dimerization between the HER3-2-2 and HER2 in CHO-HER3-2-2 cells. No pHER3 and much reduced pAKT and pERK1/2 were detected in CHO-HER3-2-2 cells. The reduced signaling activation of downstream pAKT and pERK pathways was in agreement with the lack of promotion of cell proliferation and migration in the presence and absence of NRG-1 in CHO-HER3-2-2 cells. It is interesting to note that HER2 phosphorylation in the CHO-HER3-2-2 cells was constitutively active. Since HER2 expression levels in the CHO-HER3-2-2 and CHO-HER3 cells were similar to that in the control CHO-pcDNA cells, phosphorylation of HER2 and activation of pAKT and pERK found in the CHO-HER3-2-2 should be the result of heterodimerization of the HER3-2-2 receptor with $\mathrm{CHO}$ HER2. These results suggest that over expression of HER3-2-2 somehow stimulated HER2 activation even though the PLA assay for extracellular domain association could not detect dimerization of HER3-2-2 with HER2. This disconnection of extracellular domain association and intracellular domain activation in the chimera receptor HER3-2-2 implies that the complex interaction of both extracellular fragment and intracellular domains leads to the coordinated activation of HER3/HER2 downstream signaling pathways.

Distinct activation of pAKT and pERK in the HER3/HER2 chimeric receptor implies that HER3 and HER2 cytoplasmic domains play different roles in the activation of downstream signaling pathways. Structural modeling of the intracellular kinase domain region based on a published HER3 kinase model did not reveal major structural differences between wild type HER3, HER3-2-3, and HER3-2-2 (Jura et al., 2009b; Jura et al., 2009a) (Fig. 5). Inhibition of HER3/HER2 dimerization by replacement of the HER3 TK domain with the HER2 counterpart suggests that interruption of the intracellular engagement can lead to HER3 inhibition. In addition to the kinase domain (TK), the C-terminal tail domain (CTD) of HER3 also plays a critical role in HER3 dimerization with HER2, since replacing both the TK and CTD domains with the HER2 counterparts blocked HER3 dimerization with HER2 and reduced HER3 activation and downstream signaling. This novel finding of HER3 intracellular function in its activation process provides a new strategy for perturbation of HER3 signaling activation through targeting HER3 intracellular domains in addition to the current strategy of targeting extracellular domain with monoclonal antibodies.

\section{MATERIALS AND METHODS}

\section{Cell culture and reagents}

Stable cell lines were constructed in Flp-In-CHO cells and maintained 
A

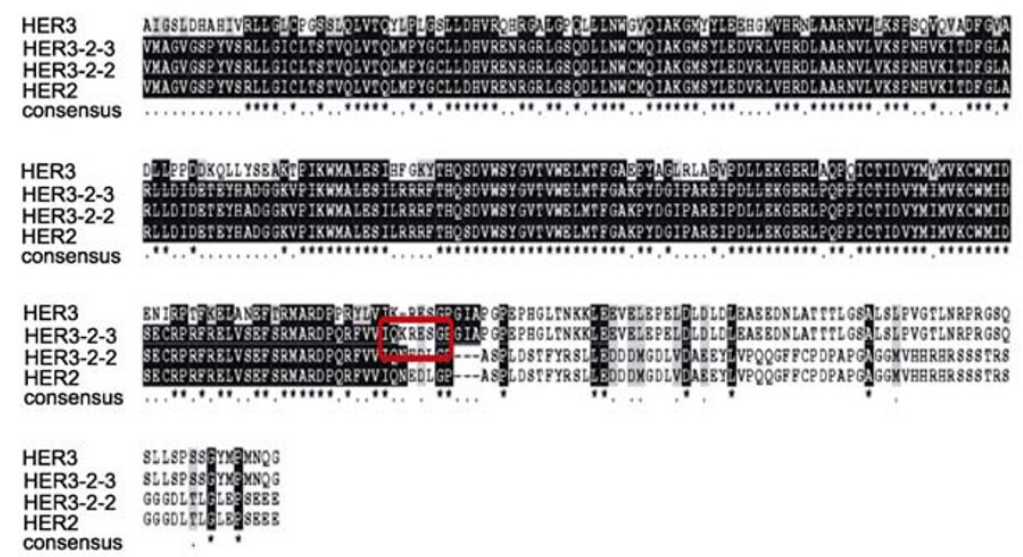

C

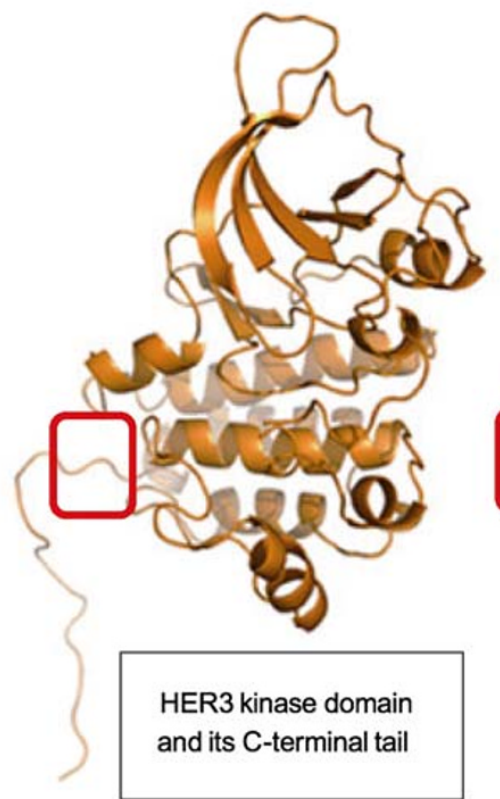

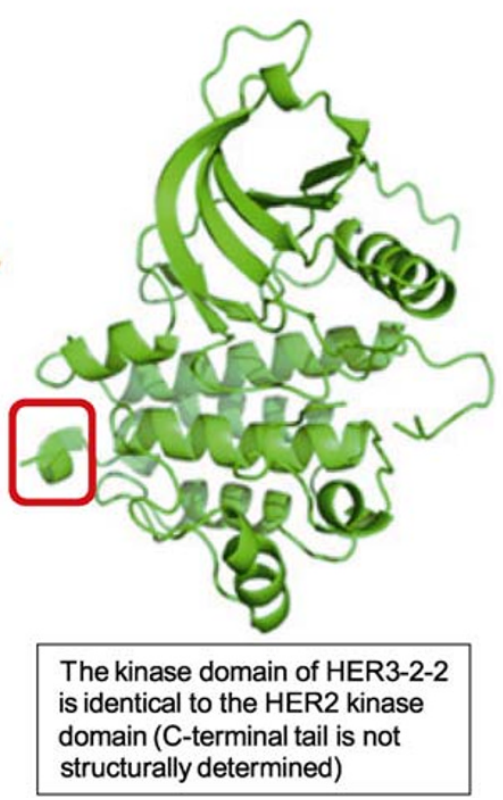

B
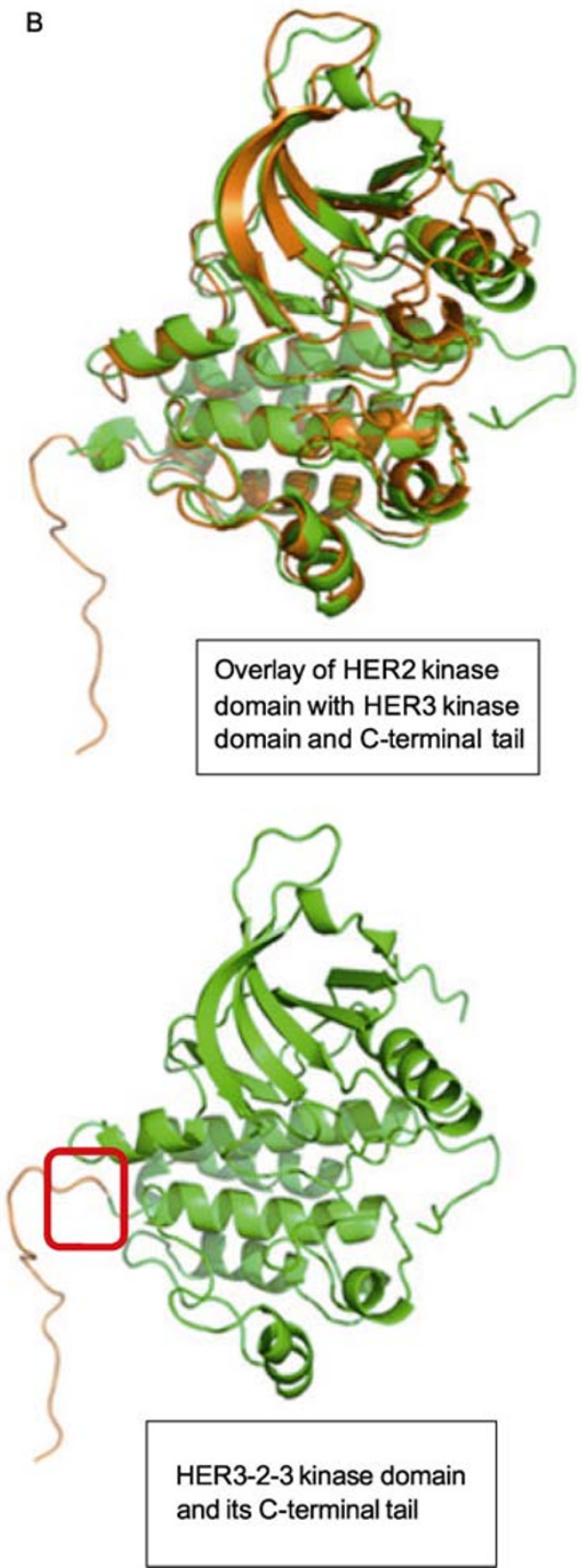

Figure 5. Sequence and structure comparisons between HER3, HER2, and HER3/2 chimeras. (A) The sequence of HER3 and HER2 are highly conserved in the kinase domain but different in their C-terminal tails. The red box indicates junction regions between the kinase domains and the C-terminal tails; (B) Structural alignment of HER3 (PDB ID 3KEX) and HER2 (PDB ID 3PP0) shows high degree of structural similarity between their kinase domains; but (C) a C-terminal $\beta$-turn is present in HER2, HER3-2-2 but not in HER3 and the predicted structure of HER3-2-3, as highlighted by the red boxes.

in Ham's F12 medium supplemented with L-glutamine (Life Technologies, Carlsbard, CA), 10\% fetal bovine serum (FBS) (HyClone, Logan, UT), and antibiotics (50 units $/ \mathrm{mL}$ penicillin and $50 \mu \mathrm{g} / \mathrm{mL}$ streptomycin, Life Technologies) in a humidified atmosphere of $5 \%$ $\mathrm{CO}_{2}$ at $37^{\circ} \mathrm{C}$. Neuregulin-1 (NRG-1) was from R\&D systems (Minneapolis, MN). Antibodies against human HER2, pHER2 pHER3, AKT, pAKT, ERK1/2 and pERK1/2 were from Epitomics (Burlingame,
CA). Antibody against HER3 (Clone 2F12) was from ThermoScientific (Rockford, IL). Antibody against $\beta$-actin was from Santa Cruz Biotechnology. HER3mAb was transiently expressed in HEK293 cells based on published amino acid sequences using a mammalian expression vector system and the antibody was purified using protein $A$ affinity resin (Fisher Scientific) as described previously (Rothe et al., 2007; Zhang et al., 2011). 


\section{Cell line construction}

Full-length human HER2 and HER3 cDNA clones were from GeneCopoeia (Rockville, MD). The HER3 constructs were made in pcDNA5/FRT/T0 and plasmids were maintained in $E$. coli strain XL1-blue (Life Technologies). HER3/HER2 chimeric receptors were constructed from PCR amplified fragments using HER2 and HER3 DNA as templates using primers listed in Table S1. Constructs were expressed in the matching CHO TREX FLIP cell line based on manufacturer's instructions.

\section{Real-time RT-qPCR}

Total RNA from cells was isolated using TRIzol reagent from Life Technologies and used as template to synthesize cDNA using iScript $^{\mathrm{TM}}$ cDNA synthesis kit (Bio-Rad, Hercules, CA). Real-time RT-PCR was performed using Ssofast ${ }^{\mathrm{TM}}$ EvaGreen ${ }^{\circledR}$ (Bio-Rad) with the oligonucleotides listed in Table S2. Real-time mRNA determinations in each stable cell line were performed with $1 \mu \mathrm{g}$ of RNA sample and normalized to $\beta$-actin using the $\Delta \Delta C_{t}$ method (Livak and Schmittgen, 2001).

\section{Flow cytometry}

Approximately $1 \times 10^{6}$ cells $/ \mathrm{mL}$ were resuspended in PBS supplemented with $2 \%$ FBS. Cells were stained with anti-HER2 (clone \# 191924, R\&D systems) or anti-HER3 antibody (clone \#66223, R\&D systems) at $4^{\circ} \mathrm{C}$ for $40 \mathrm{~min}$. Cells were washed with $4 \mathrm{~mL}$ PBS with $2 \%$ FBS, then centrifuged at $1200 \mathrm{rpm}$ for $10 \mathrm{~min}$. Supernatant was removed and cells were stained with R-PE conjugated anti-mouse IgG (Jackson ImmunoResearch Lab) at $4^{\circ} \mathrm{C}$ for $30 \mathrm{~min}$. Flow cytometry was performed using a Guava easyCyte HT (Millipore, Billerica, MA).

\section{Proximity ligation assay (PLA)}

Cells were grown in 8-well chamber slides to $80 \%$ confluency. Starved cells were treated with NRG-1 for $10 \mathrm{~min}$. Cells were then washed with PBS, fixed in $4 \%$ paraformaldehyde for $30 \mathrm{~min}$, permeabilized in $0.1 \%$ Triton X-100 for 20 min and blocked with Duolink II blocking solution (Olink Biosciences, Uppsala, Sweden). After blocking, cells were incubated with a pair of primary antibodies (anti-HER2 and anti-HER3) in a pre-heated humidity chamber for $1 \mathrm{~h}$ at $37^{\circ} \mathrm{C}$. Cells were then incubated with PLA probes diluted 1:5 in antibody diluent (Olink) in a humidified chamber for $1 \mathrm{~h}$ at $37^{\circ} \mathrm{C}$. Fluorescence images were acquired using a Zeiss Axiovert microscope (Carl Zeiss Microscopy, Thornwood, NY).

\section{Immunoprecipitation}

Treated cells were lysed with lysis buffer containing phosphatase inhibitor cocktail II and III (Sigma, St. Louis, MO) and protease inhibitor cocktail $\mathrm{V}$ (Calbiochem, San Diego, CA). Total cell lysates (1 $\mathrm{mg}$ in $1 \mathrm{~mL}$ ) were incubated with Dynabeads ${ }^{\circledR}$ protein $\mathrm{G}$ (Invitrogen) and HER3 antibody overnight at $4^{\circ} \mathrm{C}$. Captured HER3 on protein G beads were separated in a magnet stand, washed three times using PBS and dissolved in SDS sample buffer containing 2-mercaptoethanol. Proteins were resolved by $10 \%$ SDS-PAGE under reducing conditions and transferred for Western blotting.

\section{Western blot detection of HER receptors, AKT and ERK1/2 phosphorylation}

Total proteins in cell lysates were measured with BCA reagents (Thermo Scientific). The same amount of total protein $(20 \mu \mathrm{g})$ from each sample was applied to a $10 \%$ SDS-polyacrylamide gel, transferred to a nitrocellulose membrane, and blocked with $5 \%$ non-fat dry milk in TBS containing $0.1 \%$ Tween 20 (TBST) overnight at $4^{\circ} \mathrm{C}$. Blots were then incubated with primary antibodies in a dilution with TBS according to manufacturer's instructions. Blots were washed with TBST and incubated with secondary antibodies coupled with horse radish peroxidase for $30 \mathrm{~min}$. After washing three times with TBST, target proteins were detected with FluorChem $M$ imager (ProteinSimple, Santa Clara, CA) using Enhanced Chemiluminescence substrate (GE Healthcare).

\section{Cell proliferation assay}

A total of 1000 cells per well were seeded into 96-well black plates with clear bottom (Fisher Scientific) and cultured overnight at $37^{\circ} \mathrm{C}$ and $5 \%$ $\mathrm{CO}_{2}$. Treatments were applied and continued culturing for $72 \mathrm{~h}$. After 3 days of treatment, 1/10 volume of AlamarBlue ${ }^{\mathrm{TM}}$ solution (Invitrogen) was added to each well and incubated for $4 \mathrm{~h}$ at $37^{\circ} \mathrm{C}$. Fluoresecence signal was measured with excitation at $530 \mathrm{~nm}$ and emission at $590 \mathrm{~nm}$.

\section{Cell migration assay}

About $2 \times 10^{4}$ cells were seeded in the upper chamber of a 24-well Transwell plate (Corning, NY) with $0.1 \%$ BSA in serum free media and in the bottom well containing Ham's F12 media with or without $100 \mathrm{ng} / \mathrm{mL}$ NRG-1, and incubated for $18 \mathrm{~h}$ at $37^{\circ} \mathrm{C}$. Cells that migrated across the membrane were stained with $0.5 \%$ crystal violet and visualized under a microscope. The images (5-6 per well) were randomly selected and the number of cells per image was counted and plotted.

\section{Structural analysis with protein sequences}

For HER3, HER2 and HER3/2 chimeras, sequence alignment was performed using ClustalW (ch.EMBnet.org). Structure alignment of HER3 (PDB ID 3KEX) and HER2 (PDB ID 3PP0) was carried out using PyMOL (www.pymolwiki.org). The predicted structure of HER3-2-3 was constructed by fitting the kinase domain of HER2 onto the structure of HER3 kinase and tail.

\section{ACKNOWLEDGEMENTS}

The work was partially funded by grants from the Texas Emerging Technology Fund and the Welch Foundation. We thank Dr. Donna Montgomery for comments on the manuscript.

\section{ABBREVIATIONS}

AKT, acutely transforming retrovirus AKT8 in rodent T cell lymphoma; $\mathrm{CHO}$, Chinese hamster ovary; CTD, C-terminal tail; EGFR, human 
epidermal growth factor receptor; ERK1/2, extracellular signal-regulated kinase 1/2; HER2, human epidermal growth factor receptor 2; HER3, human epidermal growth factor receptor 3; MAPK, mitogen-activated protein kinase; NRG-1, neuregulin-1; pHER3, phosphorylated HER3; PI3K, phophatidylinositol-3-OH-kinase; TK, kinase domain

\section{REFERENCES}

Amin, D.N., Campbell, M.R., and Moasser, M.M. (2010a). The role of HER3, the unpretentious member of the HER family, in cancer biology and cancer therapeutics. Semin Cell Dev Biol 21, 944-950.

Amin, D.N., Sergina, N., Ahuja, D., McMahon, M., Blair, J.A., Wang, D., Hann, B., Koch, K.M., Shokat, K.M., and Moasser, M.M. (2010b). Resiliency and vulnerability in the HER2-HER3 tumorigenic driver. Sci Transl Med 2, 16ra17.

Baselga, J., and Swain, S.M. (2009). Novel anticancer targets: revisiting ERBB2 and discovering ERBB3. Nat Rev Cancer 9, 463-475.

Berger, M.B., Mendrola, J.M., and Lemmon, M.A. (2004). ErbB3/HER3 does not homodimerize upon neuregulin binding at the cell surface. FEBS Lett 569, 332-336.

Campbell, M.R., Amin, D., and Moasser, M.M. (2010). HER3 comes of age: new insights into its functions and role in signaling, tumor biology, and cancer therapy. Clin Cancer Res 16, 1373-1383.

Chan, S.D., Antoniucci, D.M., Fok, K.S., Alajoki, M.L., Harkins, R.N., Thompson, S.A., and Wada, H.G. (1995). Heregulin activation of extracellular acidification in mammary carcinoma cells is associated with expression of HER2 and HER3. J Biol Chem 270, 22608-22613.

Choi, B.-K., Fan, X., Deng, H., Zhang, N. and An, Z. (2012), ERBB3 (HER3) is a key sensor in the regulation of ERBB-mediated signaling in both low and high ERBB2 (HER2) expressing cancer cells. Cancer Medicine. doi: 10.1002/cam4.10

Engelman, J.A., Zejnullahu, K., Mitsudomi, T., Song, Y., Hyland, C., Park, J.O., Lindeman, N., Gale, C.M., Zhao, X., Christensen, J., et al. (2007). MET amplification leads to gefitinib resistance in lung cancer by activating ERBB3 signaling. Science 316, 1039-1043.

Huang, Z., Brdlik, C., Jin, P., and Shepard, H.M. (2009). A pan-HER approach for cancer therapy: background, current status and future development. Expert Opin Biol Ther 9, 97-110.

Jura, N., Shan, Y., Cao, X., Shaw, D.E., and Kuriyan, J. (2009a). Structural analysis of the catalytically inactive kinase domain of the human EGF receptor 3. Proc Natl Acad Sci U S A 106, 21608-21613.

Jura, N., Endres, N.F., Engel, K., Deindl, S., Das, R., Lamers, M.H., Wemmer, D.E., Zhang, X., and Kuriyan, J. (2009b). Mechanism for activation of the EGF receptor catalytic domain by the juxtamembrane segment. Cell 137, 1293-1307.

Kong, A., Calleja, V., Leboucher, P., Harris, A., Parker, P.J., and Larijani, B. (2008). HER2 oncogenic function escapes EGFR tyrosine kinase inhibitors via activation of alternative HER receptors in breast cancer cells. PLoS One 3, e2881.

Krug, A.W., Schuster, C., Gassner, B., Freudinger, R., Mildenberger, S., Troppmair, J., and Gekle, M. (2002). Human epidermal growth factor receptor-1 expression renders Chinese hamster ovary cells sensitive to alternative aldosterone signaling. J Biol Chem 277, 45892-45897.

Livak, K.J., and Schmittgen, T.D. (2001). Analysis of relative gene expression data using real-time quantitative PCR and the 2(-Delta Delta C(T)) Method. Methods 25, 402-408.

Mattoon, D.R., Lamothe, B., Lax, I., and Schlessinger, J. (2004). The docking protein Gab1 is the primary mediator of EGF-stimulated activation of the PI-3K/Akt cell survival pathway. BMC Biol 2, 24.

Narayan, M., Wilken, J.A., Harris, L.N., Baron, A.T., Kimbler, K.D., and Maihle, N.J. (2009). Trastuzumab-induced HER reprogramming in "resistant" breast carcinoma cells. Cancer Res 69, 2191-2194.

Rothe, M., Treder, M., Hartmann, S., Freeman, D., and Radinsky, B. (2007). Antibodies directed to HER-3 and uses thereof. In World Intellectual Property Organization, W.I.P. Organization, ed.

Schoeberl, B., Pace, E.A., Fitzgerald, J.B., Harms, B.D., Xu, L., Nie, L., Linggi, B., Kalra, A., Paragas, V., Bukhalid, R., et al. (2009). Therapeutically targeting ErbB3: a key node in ligand-induced activation of the ErbB receptor-PI3K axis. Sci Signal 2, ra31.

Shepard, H.M., Brdlik, C., and Schreiber, H. (2008). Signal integration: a framework for understanding the efficacy of therapeutics targeting the human EGFR family. The Journal of Clinical Investigation 118, 3574-3581.

Sierke, S.L., Cheng, K., Kim, H.H., and Koland, J.G. (1997). Biochemical characterization of the protein tyrosine kinase homology domain of the ErbB3 (HER3) receptor protein. Biochem J 322 (Pt 3), 757-763.

Suenaga, A., Takada, N., Hatakeyama, M., Ichikawa, M., Yu, X., Tomii, K., Okimoto, N., Futatsugi, N., Narumi, T., Shirouzu, M., et al. (2005). Novel mechanism of interaction of p85 subunit of phosphatidylinositol 3-kinase and ErbB3 receptor-derived phosphotyrosyl peptides. J Biol Chem 280, 1321-1326.

Wheeler, D.L., Huang, S., Kruser, T.J., Nechrebecki, M.M., Armstrong, E.A., Benavente, S., Gondi, V., Hsu, K.T., and Harari, P.M. (2008). Mechanisms of acquired resistance to cetuximab: role of HER (ErbB) family members. Oncogene 27, 3944-3956.

Zhang, N., Liu, L., Dan Dumitru, C., Cummings, N.R., Cukan, M., Jiang, Y., Li, Y., Li, F., Mitchell, T., Mallem, M.R., et al. (2011). Glycoengineered Pichia produced anti-HER2 is comparable to trastuzumab in preclinical study. MAbs 3, 298-98. 